\title{
Workshop on Liaison Psychiatry
}

The College Liaison Psychiatry Group held its first one-day workshop at the Society of the Chemical Industry on 29 November 1985. The workshop was attended by approximately 70 members and was organised on behalf of the Group by Bryan Lask, Hospital for Sick Children, London. The aims were to provide a forum to discuss the practical issues involved in liaison psychiatry so that clinicians might learn how to evolve the most suitable form of practice according to local demands and resources.

Three papers were presented during the morning session. The first, by Peter Maguire of University Hospital of South Manchester, was concerned with the establishment of a liaison service for a particular group of patients. He took as his model the service which has been developed in Manchester for women who have undergone mastectomy and there was particular emphasis on the role of the nurse in detecting psychiatric morbidity and providing subsequent counselling. Sandy Cooper of Leverndale and Victoria Hospitals, Glasgow, presented a paper in which he reviewed the obstacles which have to be faced when establishing a liaison service and he presented figures from his own service showing how the referral rate rises once a systematic service is established under responsibility of one consultant. Finally, Robert Tattersall, a consultant physician at Queen's Medical Centre, Nottingham, outlined his views of what was required of psychiatry from a physician's standpoint.
His remarks were both witty and provocative but he made a clear plea for closer collaboration between psychiatry and general medicine, particularly at a consultant level and he listed several important areas of medical practice in which he felt psychiatry could make a greater contribution than it does at present.

During the afternoon session members divided into four groups for informal discussions and there was finally a panel discussion, chaired by Joseph Connolly, Maudsley Hospital, London, in which there was ample opportunity to discuss the scope of liaison psychiatry and different models of practice. There were clear differences in emphasis between various participants and there was what politicians would call a frank exchange of views between those who viewed liaison psychiatry as being concerned essentially with the treatment of psychiatric illness in a general hospital setting and others who saw liaison psychiatry as having additional responsibilities of managing medical patients who were not strictly psychiatrically ill.

The Workshop, which was attended by members from all over the United Kingdom, was considered a successful first venture for the Group and it is hoped it will be the first of several such occasions.

GeofrRey LLOYD Secretary, Liaison Psychiatry Group

\section{Correspondence}

\section{Overseas candidates and psychiatric examinations}

\section{Dear Sirs}

As teachers of psychiatry concerned with the education of indigenous and overseas doctors, we would like to add our comments to the debate (Bulletin, March 1986, 10, 59-63) concerning the relative failure rates of these groups in the MRCPsych examination.

As well as helping candidates, including many from overseas, prepare for the Membership examination, we are involved in teaching examination technique to candidates taking the Diploma of Psychiatry awarded by the Institute of Psychiatry. The diploma is of equivalent standard to the Membership but is specifically designed to meet the needs of doctors returning to practise in their native countries. Thus, in addition to the clinical and basic science necessary for psychiatry, there is an emphasis on neurology and Third
World psychiatry, whilst less relevant areas, such as British Mental Health legislation, are not included. Like the MRCPsych there are two parts to the Diploma, Part A consisting of a short answer question paper in the sciences basic to psychiatry and neorology, and Part B including clinical examinations in neurology and psychiatry as well as vivas, and MCQs and SAQs (but not essays). There is also a minimum requirement of study and practice of psychiatry in the UK before entry to the examination though GMC registration is not required. Unlike the Membership the issue of possible examiner bias favouring indigenous candidates does not arise, since all candidates are from abroad.

Since the Diploma was introduced in 1983, 74 candidates have sat Part A, and 65 candidates Part B. The majority come from the Indian subcontinent (36\%) and the Middle East $(30 \%)$, although doctors from Sudan, other parts of Africa, Far East, South America and Europe have also passed the Diploma examinations; $70 \%$ of candidates were successful at the first attempt at Part $A$ and $12 \%$ at their second, while $54 \%$ were successful at their first attempt at Part B; 72\% of those sitting Part B of the Diploma successfully passed the examination after two attempts. As the Diploma was only recently introduced as a post-graduate qualification in psychiatry, the numbers who have sat the examination are still relatively small. However, there is little 
evidence that Indian candidates do worse than other groups. (See Table).

TABLE

Successes in Parts A and B of the Diploma of Psychiatry awarded by the Institute of Psychiatry

\begin{tabular}{lcc}
\hline Part A & & \\
Country of Origin & No & $\begin{array}{c}\text { Passes Ist and } \\
\text { 2nd attempt \% }\end{array}$ \\
\hline Africa (exclud. Sudan) & 7 & $5(71 \%)$ \\
Sudan & 8 & $8(100 \%)$ \\
Indian Sub-Continent & 27 & $21(78 \%)$ \\
Middle East & 22 & $20(91 \%)$ \\
Far East & 4 & $3(75 \%)$ \\
S America/Europe & 6 & $5(83 \%)$ \\
& & \\
Part B & & \\
Africa (exclud. Sudan) & 5 & $3(60 \%)$ \\
Sudan & 9 & $7(78 \%)$ \\
Indian Sub-Continent & 19 & $12(63 \%)$ \\
Middle East & 23 & $17(74 \%)$ \\
Far East & 5 & $4(80 \%)$ \\
S America & 4 & $4(100 \%)$ \\
\hline
\end{tabular}

Like the Membership, the majority of those who fail the Diploma (Part B) do so in more than one part of the examination.

Clearly, there are many reasons why candidates fail post-graduate examinations in this country and the College now provides an excellent system for providing individual feed-back for failure in the Membership; a service which we believe is unique among the Royal Colleges. We would also strongly support the recommendation of the Collegiate Trainees Committee that greater advantage is taken of this. However, based on this Institute's wide teaching experience with examination candidates from many different countries, we would like to make the following general observations concerning the reasons for failure that apply more specifically to doctors from abroad and which are not simply due to poor command of the English language.

First, some candidates seem unfamiliar with examination methods in this country. There are still a surprising number of candidates who are unable to deal with the common type of essay or viva question that requires discussion or application of knowledge rather than mere repetition of facts. Consequently, such candidates may have adequate 'book' knowledge, but fail for not having answered the question that has been set in the case of the essay or who are 'thrown' by the case vignette style of viva question.

Secondly, the clinical examination is still a great obstacle for many. Candidates often have the impression that something novel is required of them and that the examination is in some way very different to ward patient management with which they are familiar. Although by its very nature the examination setting is artificial, demonstration of the candidate's clinical competence to take charge of patients is the prime issue, and this must be demonstrated to the examiners. Frequently the problem is that trainees are not required to assess patients adequately in their 'ward round' presentations, and restrict themselves to factual presentations, leaving the thinking and planning to be done by senior colleagues. Training schemes need to foster the regular presentations of patients at case conferences on ward rounds, with the trainees being asked to formulate their assessment in the manner required under examination conditions. Although the overconfident candidate may fail to impress, one of the major difficulties for overseas candidates, in our view, is lack of confidence in the case assessment, and this can be compounded with increasing number of attempts at the examination.

The solution is for the candidate to spend less time reading books and more time practising examination technique with colleagues (preferably senior). As well as practising essays, MCQs and 'mock' vivas (with emphasis on the 'case vignette' style of question) we advise that candidates should clerk at least 10 common psychiatric cases under examination conditions by allowing themselves only one hour to clerk and prepare an assessment on a patient not known by them. The case should then be presented to a colleague in the correct examination format in $\mathbf{3 0}$ minutes (i.e. assessment including salient features of the history, mental state findings, differential diagnosis, possible aetiological factors, followed by management and prognosis). Attending a course in examination technique may help, but cannot completely replace the need for individual practice and thorough familiarity with the examination format.

Finally, we would like to emphasise that the above comments are our own and that we are not representing the views of either the Institute of Psychiatry or the Royal College of Psychiatrists. However, we hope that they may be of help to those who are sitting psychiatric examinations in this country.

Institute of Psychiatry

ANNE FARMER

NeIL HOLDEN

Denmark Hill, London SES

\section{Dear Sirs}

As a psychiatrist involved with a Postgraduate Teaching Programme designed to help junior doctors with their MRCPsych examination I am aware that of the total number of doctors who sit their examination each year the pass rate is considerably higher among the British doctors than among those for whom English is a second language.

One of the reasons for this, in my opinion, is that in the multiple choice questions certain words are used which are difficult to evaluate by the foreign doctors e.g. commonly, frequently, significantly, occasionally. It would be helpful to the foreign candidates if a rough guide as to what is expected in percentages was to be given for these and similar 